\title{
A MINIMUM TRAPPING TIME PROBLEM IN OPTIMAL CONTROL THEORY
}

\author{
M. E. FISHER, J. L. NOAKES AND K. L. TEO ${ }^{1}$
}

(Received 11 May 1989)

\begin{abstract}
In this paper we consider a natural extension of the minimum time problem in optimal control theory which we refer to as the minimum trapping time problem. The minimum trapping time problem requires a fixed time interval $[0, T]$, where $T$ is finite. The aim is to determine a control for which the system trajectory not only reaches a specified target in minimum time but also remains trapped within the target until time $T$. Our aim is to devise a computational procedure for solving the minimum trapping time problem. The computational procedure we adopt uses control parametrisation in which the class of controls is approximated by a class of piecewise constant functions. The problem we are solving is therefore an approximation to the original minimum trapping time problem. Some properties for the approximate problem are then established. These lead to an extremely efficient iterative procedure for calculating the minimum trapping time.
\end{abstract}

\section{Introduction}

An extremely important and extensively studied problem in optimal control theory is the minimum time problem (see, for example, $[1,2,3,4]$ ). In this paper we consider a natural extension of this which we refer to as the minimum trapping time problem. We are interested in an actual solution procedure for solving problems which can be formulated as minimum trapping time problems. To our knowledge, problems of this type have not been formulated previously in the optimal control literature, much less solved.

The minimum trapping time problem requires a fixed time interval $[0, T]$, where $T$ is finite. The aim is to determine a control, subject to its constraints, for which the system trajectory not only reaches a specified target in minimum

\footnotetext{
'Department of Mathematics, The University of Western Australia, Western Australia 6009.

(C) Copyright Australian Mathematical Society 1990, Serial-fee code 0334-2700/90
} 
time but also remains trapped within the target until time $T$. Of course, a necessary condition for such a minimum time, called $\tau^{*}$, to exist is that the system is controllable to the target in time $t<T$.

Many problems in optimal control can be naturally formulated as minimum trapping time problems. There are biological or biomedical problems, for example, where it may be desirable to quickly return one or more of the states to acceptable levels and keep them there. A specific example might be to use externally supplied insulin to control a diabetic patient's blood glucose level. Another class of problems for which the minimum trapping problem is a natural setting is that of tracking problems in which the aim is to reach and remain close to a desirable trajectory in minimum time.

In Section 2, there is a precise statement of the minimum trapping time problem and various assumptions which guarantee the existence of a solution. The actual computation of the minimum trapping time requires that the class of admissible controls be approximated. As discussed in Section 3 , the computational procedure we adopt uses control parametrisation (see $[5,6,7])$ in which the class of controls is approximated by a class of piecewise constant functions. The problem we are solving is therefore an approximation to the original minimum trapping time problem. Some properties for the approximate problem are then established. These lead to an extremely efficient iterative method for calculating the minimum trapping time as discussed in Section 4. Two simple examples are then presented in Section 5 to illustrate the implementation of the solution procedure.

\section{Problem statement}

Consider the following differential equation on the fixed time interval $[0, T]$ :

$$
\dot{x}(t)=f(t, x(t), u(t)) .
$$

Here, $x \equiv\left[x_{1}, \ldots x_{n}\right]^{\top}, u \equiv\left[u_{1}, \ldots, u_{r}\right]^{\top}$ are, respectively, the state and control vectors and $f \equiv\left[f_{1}, \ldots, f_{n}\right]^{\top}$. The initial condition for the differential equation (2.1a) is

$$
x(0)=x^{0},
$$

where $x^{0}$ is a given vector in $\mathbf{R}^{n}$. Define

$$
U=\left\{v \in R^{r}:\left(E^{i}\right)^{\top} v \leq b_{i}, i=1, \ldots, q\right\},
$$

where $E^{i}, i=1, \ldots, q$, are $r$-vectors, and $b_{i}, i=1, \ldots, q$, are real numbers. A bounded measurable function from $[0, T]$ into $U$ is called an admissible control. Let $\mathscr{U}$ be the class of all such admissible controls and 
for each $u \in \mathscr{U}$, let $x(\cdot \mid u)$ be the corresponding solution of the system (2.1). We define a target set $Z$ by the inequality

$$
g(t, x) \leq 0,
$$

where $g$ is a real-valued function defined on $[0, T] \times R^{n}$.

The problem is to find the minimum time $\tau^{*} \in(0, T)$ and a corresponding control $u^{*}$ for the solution $x\left(t \mid u^{*}\right)$ to system (2.1) to reach and remain in the targei sei $Z$ untîl time $T$. We căl this the miñimim trappping time problem and refer to it as problem $(P)$. To be more specific, let $\mathscr{F}$ be a subset of $[0, T]$ defined as follows: Corresponding to each $\tau \in \mathscr{F}$, there exists a control $u \in \mathscr{U}$ such that $g(t, x(t \mid u)) \leq 0$, for all $t \in[\tau, T]$. Our problem is to find a $\tau^{*} \in(0, T)$ such that

$$
\tau^{*}=\inf _{\tau \in \mathscr{T}} \tau \text {. }
$$

$\tau^{*}$ will be called the minimum trapping time.

To solve our problem we assume the following.

(A1) For each compact set $\Omega \subset R^{r}$, there exists a positive constant $K$ such that $|f(t, x, u)| \leq K(1+|x|)$, for all $(t, x, u) \in[0, T] \times R^{n} \times \Omega$;

(A2) $f_{i}(t, x, u), i=1, \ldots, n$, are piecewise continuous on $[0, T]$ for each $(x, u) \in R^{n} \times R^{r}$; furthermore, $f_{i}(t, x, u), i=1, \ldots, n$, are continuously differentiable with respect to each of the components $x, u$ for each fixed $t \in[0, T]$;

(A3) $g(t, x)$ is continuously differentiable with respect to each of the components $t, x$;

(A4) There exists $\tau \in(0, T)$ such that $\tau \in \mathscr{F}$. (A necessary condition for this assumption to be satisfied is that there exists $t \in(0, T)$ such that the system (2.1) is controllable to the target in time $t$.)

(A5) Let $\tau \in \mathscr{F}$ and $\varepsilon>0$ be arbitrary such that $\tau+\varepsilon \leq T$. Then, there exists $u \in \mathscr{U}$ such that $g(t, x(t \mid u))<0$ for all $t \in[\tau+\varepsilon, T]$ where $x(t \mid u)$ satisfies (2.1).

(A6) $\mathscr{F}$ is closed.

(A7) $x^{0} \notin Z$.

As a consequence of the definition of $\mathscr{F}$ and assumptions (A4) and (A6) we have that $\mathscr{F}=\left[\tau^{*}, T\right]$ where $\tau^{*} \in(0, T)$.

In this paper, our aim is to derive an implementable algorithm for solving the minimum trapping time problem $(P)$. To this end, consider the following optimal control problem which we refer to as $\left(P_{\tau}\right)$ :

For a fixed $\tau \in[0, T]$ minimise the functional $J(\tau, u)$ with respect to $u \in \mathscr{U}$ where

$$
J(\tau, u)=\int_{\tau}^{T}\{\max (g(t, x(t \mid u)), 0)\}^{2} d t,
$$

and $x(t \mid u)$ satisfies the system (2.1). 
Denote the optimal value of the objective for this problem as $\psi(\tau)$; that is,

$$
\psi(\tau)=\min _{u \in \mathscr{U}} J(\tau, u)
$$

Note that $\psi(\tau)=0$ if and only if $\tau \in \mathscr{F}$. One obvious property of the real valued function $\psi$ is that it is a nonincreasing function of $\tau$. The solution $\tau^{*}$ to the minimum trapping time problem $(P)$, if it exists, is then the minimum value of $\tau \in[0, T]$ which is a zero of the function $\psi$.

The problem we now have is essentially to find the first zero of the real valued function $\psi$ in $[0, T]$. One approach is to use a zero-finding algorithm to generate a sequence of points $\tau_{k}, k=1,2, \ldots$ which, hopefully, will then converge to $\tau^{*}$. At each $\tau_{k}$ we evaluate $\psi\left(\tau_{k}\right)$ by solving the optimal control problem $\left(P_{\tau_{k}}\right)$.

In the absence of any knowledge of the properties of $\psi$, other than the existence of a zero in $[0, T]$, the obvious method to use is the bisection method. In 10 iterations, this method will provide us with an interval containing $\tau^{*}$ which is $0.1 \%$ the size of $[0, T]$. There are of course other methods based on certain assumptions about the form of the function $\psi$ near $\tau^{*}$ which may provide this accuracy in fewer iterations. These methods all require the solution of a sequence of optimal control problems $\left(P_{\tau}\right)$ in order to evaluate function values of $\psi$. The method we use to solve these subproblems is based on the concept of control parametrisation $([5,6,7])$ in which the time interval $[0, T]$ is partitioned into equal subintervals on which the controls are approximated by constant functions. Note that any member in the family of gradient-restoration methods due to Miele and his coworkers $([8,9,10])$ could also be used to solve these subproblems. In the next section we use control parametrisation and derive, under certain assumptions, properties of the function $\psi$ which enable us to construct a simple but extremely efficient algorithm for estimating $\tau^{*}$.

\section{Control parametrisation}

To begin, partition the time interval $[0, T]$ into $N_{p}$ equal subintervals of length $\Delta=T / N_{p}$ which are closed on the left and open on the right. On each subinterval, each control is approximated by a constant function. Hence we have:

$$
u(t)=\sum_{l=1}^{N_{p}} \sigma^{p} \chi_{l}(t)
$$


where $\chi_{1}(t)$ is the indicator function defined by

$$
\chi_{l}(t)= \begin{cases}1 & (l-1) \Delta \leq t<l \Delta, \\ 0 & \text { elsewhere }\end{cases}
$$

and $\sigma^{p}$ is a constant vector in $R^{s}$ with $s=r N_{p}$. Let $A^{p}$ be the class of all those piecewise constant functions which are consistent with the partition, namely, $u^{p} \in A^{p}$ can be written as

$$
u^{p}(t)=\sum_{l=1}^{N_{p}} \sigma^{p, l} \chi_{l}(t)
$$

where $\sigma^{p, l} \in R^{r}$. This means that each control $u^{p} \in A^{p}$ can be identified with the vector $\sigma^{p}=\left[\left(\sigma^{p, 1}\right)^{\top}, \ldots,\left(\sigma^{p, N_{p}}\right)^{\top}\right]^{\top}$ where, for $l=1, \ldots, N_{p}$, $\left.\sigma^{p, l}=\mathrm{]} \sigma_{1}^{p, l}, \ldots, \sigma_{r}^{p, l}\right]^{\top}$. For convenience, we will sometimes write $\sigma^{p}$ in the form $\left[\sigma_{1}^{p}, \ldots, \sigma_{s}^{p}\right]^{\top}$.

Now define

$$
U^{p}=U \cap A^{p}
$$

and

$$
\Sigma^{p}=\left\{\sigma^{p} \in R^{s}:\left(E^{i}\right)^{\top} \sigma^{p, l} \leq b_{i}, l=1, \ldots, N_{p}, i=1, \ldots, q\right\} .
$$

Let $x\left(\cdot \mid \sigma^{p}\right)$ represent the solution of the system (2.1) corresponding to the control $\sigma^{p} \in \Sigma^{p}$ and let $F^{p}$ be a subset of $F$ in which the controls are restricted to $U^{p}$.

For a given partition of $[0, T]$, we obtain an approximation to the optimal control problem $\left(P_{\tau}\right)$ by restricting the control to $U^{p}$. The approximate problem will be formulated as follows: For a fixed $\tau \in[0, T]$ minimise the function $J^{p}\left(\tau, \sigma^{p}\right)$ with respect to $\sigma^{p} \in \Sigma^{p}$ where

$$
J^{p}\left(\tau, \sigma^{p}\right)=\int_{\tau}^{T}\left\{\max \left(g\left(t, x\left(t \mid \sigma^{p}\right)\right), 0\right)\right\}^{2} d t,
$$

and $x\left(t \mid \sigma^{p}\right)$ satisfies the system (2.1).

We denote the optimal value of the objective for this optimisation problem as $\psi^{p}(\tau)$, that is,

$$
\psi^{p}(\tau)=\min _{\sigma^{p} \in \Sigma^{p}} J^{p}\left(\tau, \sigma^{p}\right)
$$

This optimal parameter selection problem will be referred to as $\left(P_{p, \tau}\right)$.

Lemma 3.1. There exists $a \hat{\tau} \in(0, T)$ and a positive integer $\hat{p}$ such that $t \in F^{p}$ for all $t \in(\hat{\tau}, T]$ and all $p>\hat{p}$.

Proof. By (A4) and (A5), there exists a $\hat{\tau} \in(0, T)$ and a $\widehat{u} \in U$ such that $g(t, x(t \mid \widehat{u}))<0$ for all $t \in(\widehat{\tau}, T]$. From Lemma 4.3 in [11], $x\left(t \mid u^{p}\right)$, 
where $u^{p} \in U^{p}$, can be made arbitrarily close to $x(t \mid u)$, where $u \in U$, for all $t \in[0, T]$ provided $p$ is large enough. It follows from (A3), therefore, that there exists $\hat{p}$ such that $g\left(t, x\left(t \mid u^{p}\right)\right) \leq 0$ for all $t \in(\widehat{\tau}, T]$ and all $p>\widehat{p}$.

As in (2.4), we define $\tau^{p, *}$ by

$$
\tau^{p, *}=\inf _{\tau \in F^{p}} \tau,
$$

and denote by $\sigma^{p, *}$ the corresponding value of $\sigma^{p}$ which minimises $J^{p}$. If we repeat assumption (A6) for $F^{p}$ then $F^{p}=\left[\tau^{p, *}, T\right]$ where $\tau^{p, *} \in$ $(0, T)$.

The following Lemma lists some properties of the function $\psi^{p}:[0, T] \rightarrow$ $[0, \infty]$ which are immediate consequences of our definitions and assumptions (A1) to (A7).

LEMMA 3.2. For a fuxed positive integer $p$,

(i) $\psi^{p}:[0, T] \rightarrow[0, \infty]$ is nonincreasing;

(ii) $\psi^{p}(0)>0$ and $\psi^{p}(T)=0$;

(iii) $g\left(\tau^{p, *}, x\left(\tau^{p, *} \mid \sigma^{p, *}\right)\right)=0$.

We now make an assumption regarding the manner in which the system trajectories enter the target set $Z$. In most cases this is equivalent to saying that, at the minimum trapping time, the system trajectories cross the boundary of the target set transversely.

(A8) Let $h:[0, T] \times \Sigma^{p} \rightarrow R$ be defined near $\left(\tau^{p, *}, \sigma^{p, *}\right)$ by

$$
h\left(\tau, \sigma^{p}\right)=g\left(\tau, x\left(\tau \mid \sigma^{p}\right)\right) ;
$$

then $(\partial h / \partial \tau)\left(\tau^{p, *}, \sigma^{p, *}\right) \neq 0$.

LEMMA 3.3. There exists $N \subset \Sigma^{p}$ and a unique $C^{1}$ function $\phi: N \rightarrow[0, T]$ with $\varphi\left(\sigma^{p, *}\right)=\tau^{p, *}$ such that

$$
g\left(\varphi\left(\sigma^{p}\right), x\left(\varphi\left(\sigma^{p}\right) \mid \sigma^{p}\right)\right)=0
$$

and

$$
\lim _{r \rightarrow \varphi\left(\sigma^{p}\right)} \frac{\partial J^{p}}{\partial \tau}\left(\tau, \sigma^{p}\right)=0
$$

$$
\lim _{r \rightarrow \varphi\left(\sigma^{p}\right)} \frac{\partial^{2} J^{p}}{\partial \tau^{2}}\left(\tau, \sigma^{p}\right)=0
$$


(iii)

$$
\lim _{r \rightarrow \varphi\left(\sigma^{p}\right)} \frac{\partial^{2} J^{p}}{\partial \sigma_{j}^{p} \partial \tau}\left(\tau, \sigma^{p}\right)=0, j=1, \ldots, s .
$$

Proof. The implicit function theorem together with the hypothesis (A8) implies the existence of a neighbourhood $N$ of $\sigma^{p, *}$ in $\Sigma^{p}$ and a unique $C^{1}$ function $\varphi: N \rightarrow[0, T]$ satisfying $\varphi\left(\sigma^{p, *}\right)=\tau^{p, *}$ and $h\left(\varphi\left(\sigma^{p}\right), \sigma^{p}\right)=$ 0 . This establishes (3.9). From (A8) it follows that $g\left(\tau, x\left(\tau \mid \sigma^{p}\right)\right) \geq 0$ for $\tau \leq \varphi\left(\sigma^{p}\right)$ and sufficiently close. Therefore, and by (3.5), $\partial J^{p} / \partial \tau=$ $-\left\{g\left(\tau, x\left(\tau \mid \sigma^{p}\right)\right)\right\}^{2}$ for $\tau<\varphi\left(\sigma^{p}\right)$. But $J^{p}\left(\tau, \sigma^{p}\right)=0$, for $\tau>\varphi\left(\sigma^{p}\right)$, and this, together with (3.9), establishes equations (3.10) to (3.12).

We next impose a smooth selection hypothesis: (A9) There exists a $C^{1}$ function $\xi:\left[0, \tau^{p, *}\right] \rightarrow \Sigma^{p}$ such that

(i) $\xi\left(\tau^{p, *}\right)=\sigma^{p, *}$ and

(ii) $\psi^{p}(\tau)=J^{p}(\tau, \xi(\tau))$ for $\tau<\tau^{p, *}$.

Our aim is ultimately to approximate $\psi^{p}$ by a polynomial near $\left(\tau^{p, *}, \sigma^{p, *}\right)$. However, it is possible that $\psi^{p}$ may not even be $C^{2}$ and so we first consider how to approximate it by a $C^{2}$ function $\widehat{\psi}^{p}$ in a neighbourhood of $\left(\tau^{p, *}, \sigma^{p, *}\right)$. This can be achieved by approximating $J^{p}$ by a sufficiently smooth function $\widehat{J}^{p}$ and then defining $\widehat{\psi}^{p}(\tau)=\widehat{J}^{p}(\tau, \xi(\tau)) . J^{p}$ is approximated by $\widehat{J}^{p}$ in such a way that the partial derivatives of $\widehat{J}^{p}$ are the same as those of $J^{p}$, at least where the latter are defined, namely

$$
\widehat{J}^{p}\left(\tau^{p, *}, \sigma^{p, *}\right)=0
$$

$$
\frac{\partial \widehat{J}^{p}}{\partial \tau}\left(\varphi\left(\sigma^{p}\right), \sigma^{p}\right)=0
$$

(ii)

$$
\frac{\partial^{2} \widehat{J}^{p}}{\partial \tau^{2}}\left(\varphi\left(\sigma^{p}\right), \sigma^{p}\right)=0
$$

$$
\frac{\partial^{2} \widehat{J}^{p}}{\partial \sigma_{j}^{p} \partial \tau}\left(\varphi\left(\sigma^{p}\right), \sigma^{p}\right)=0, \quad j=1, \ldots, s .
$$

We also require that, for a given value of $\tau, \widehat{J}^{p}\left(\tau, \sigma^{p}\right)$ should be minimised by taking $\sigma^{p}=\xi(\tau)$. For a fixed value of $\tau$ we now expand $\widehat{J}^{p}$ in a Taylor expansion in $\sigma^{p}$ about $\xi(\tau)$. There exists a neighbourhood $N$ of $\sigma^{p, *}$ in 
$\Sigma^{p}$ and functions $c_{i, j}: N \times\left[0, \tau^{p, *}\right] \rightarrow R$ with $c_{i, j}=c_{j, i}$ such that

$$
\begin{aligned}
\widehat{J}^{p}\left(\tau, \sigma^{p}\right)=\widehat{\psi}^{p}(\tau) & +\sum_{i=1}^{s} \frac{\partial \widehat{J}^{p}}{\partial \sigma_{i}^{p}}(\tau, \xi(\tau))\left(\sigma_{i}^{p}-\xi(\tau)_{i}\right) \\
& +\sum_{i=1}^{s} \sum_{j=1}^{s}\left(\sigma_{i}^{p}-\xi(\tau)_{i}\right) c_{i, j}\left(\tau, \sigma^{p}\right)\left(\sigma_{j}^{p}-\xi(\tau)_{j}\right)
\end{aligned}
$$

We shall assume that the $c_{i, j}$ are $C^{2}$ functions.

LemMA 3.4. At the point $\tau=\tau^{p, *}$, we have, for $i=1,2, \ldots, s$,

$$
\sum_{i=1}^{s} \frac{\partial \widehat{J}^{p}}{\partial \sigma_{i}^{p}}\left(\tau^{p, *}, \xi\left(\tau^{p, *}\right)\right) \xi^{\prime}\left(\tau^{p, *}\right)_{i}=0
$$

and

$$
\sum_{i=1}^{s} \frac{\partial \widehat{J}^{p}}{\partial \sigma_{i}^{p}}\left(\tau^{p, *}, \xi\left(\tau^{p, *}\right)\right) \xi^{\prime \prime}\left(\tau^{p, *}\right)_{i}=0
$$

Proof. Let $D_{\sigma^{p}} \widehat{J}^{p}\left(\tau^{p, *}, \xi\left(\tau^{p, *}\right)\right)$ be the vector in $R^{s}$ with components

$$
\frac{\partial \widehat{J}^{p}}{\partial \sigma_{i}^{p}}\left(\tau^{p, *}, \xi\left(\tau^{p, *}\right)\right), i=1, \ldots, s .
$$

Consider a neighbourhood $N$ of $\sigma^{p, *}$ in $\Sigma^{p}$ in which $\widehat{J}^{p}\left(\tau^{p, *}, \sigma^{p}\right)$ is minimised by taking $\sigma^{p}=\xi\left(\tau^{p, *}\right)$. If $\xi\left(\tau^{p, *}\right)$ is an interior point of $\Sigma^{p}$ then $D_{a^{p}} \widehat{J}^{p}\left(\tau^{p, *}, \xi\left(\tau^{p, *}\right)\right)=0$ and (3.18) and (3.19) follow. Suppose $\xi\left(\tau^{p, *}\right)$ is a boundary point of $\Sigma^{p}$ lying on exactly one of the hyperplanes which form the boundary of $\Sigma^{p}$ as defined by (3.4). Suppose that

$$
D_{\sigma^{p}} \widehat{J}^{p}\left(\tau^{p, *}, \xi\left(\tau^{p, *}\right)\right) \neq 0 .
$$

A necessary condition that $\sigma^{p}=\xi\left(\tau^{p, *}\right)$ is a minimum point of $\widehat{J}^{p}\left(\tau^{p, *}, \sigma^{p}\right)$ is that $D_{\sigma^{p}} \widehat{J}^{p}\left(\tau^{p, *}, \xi\left(\tau^{p, *}\right)\right)$ is a vector orthogonal to the hyperplane containing $\xi\left(\tau^{p, *}\right)$. Let $\tau$ be close to $\tau^{p, *}$. Since $\widehat{J}^{p}$ is $C^{1}$ there exists a neighbourhood $N^{\prime}$ of $\sigma^{p, *}$ in $\Sigma^{p}$ in which $D_{\sigma^{p}} \widehat{J}^{p}(\tau, \xi(\tau)) \neq 0$ and $\widehat{J}^{p}\left(\tau, \sigma^{p}\right)$ is minimized by taking $\sigma^{p}=\xi(\tau)$. Hence $\xi(\tau)$ must also be on the same hyperplane forming part of the boundary of $\Sigma^{p}$ as $\xi\left(\tau^{p, *}\right)$ since, if not, $\xi(\tau)$ would be an interior point of $\Sigma^{p}$ which contradicts (3.20). This implies that $\xi^{\prime}\left(\tau^{p, *}\right)$ is a vector in the same hyperplane. This argument can be repeated to show that $\xi^{\prime \prime}\left(\tau^{p, *}\right)$ is also a vector in this hyperplane. Hence, $\xi^{\prime}$ and $\xi^{\prime \prime}$ are vectors orthogonal to $D_{\sigma^{p}} \widehat{J}^{p}$ at $\tau=\tau^{p, *}$. A similar argument can be presented in the case when $\xi\left(\tau^{p, *}\right)$ lies on the intersection of two or more hyperplanes which form the boundary of $\Sigma^{p}$ to show that $\xi^{\prime}\left(\tau^{p, *}\right)$ (and 
$\left.\xi^{\prime \prime}\left(\tau^{p, *}\right)\right)$ is again a vector orthogonal to $D_{\sigma^{p}} \widehat{J}^{p}\left(\tau^{p, *}, \xi\left(\tau^{p, *}\right)\right)$. This proves (3.18) and (3.19).

THEOREM 3.1. The $C^{2}$ function $\widehat{\psi}^{p}$ satisfies

$$
\widehat{\psi}^{p}\left(\tau^{p, *}\right)=\left(\widehat{\psi}^{p}\right)^{\prime}\left(\tau^{p, *}\right)=\left(\widehat{\psi}^{p}\right)^{\prime \prime}\left(\tau^{p, *}\right)=0
$$

Proof. $\widehat{\psi}^{p}\left(\tau^{p, *}\right)=0$ because of $(3.13)$ and $\xi\left(\tau^{p, *}\right)=\sigma^{p, *} .\left(\widehat{\psi}^{p}\right)^{\prime}\left(\tau^{p, *}\right)=$ 0 from (3.14) and (3.18), where we have again used $\xi\left(\tau^{p, *}\right)=\sigma^{p, *}$. Finally, from (3.15), (3.17), and (3.19),

$$
\begin{aligned}
0 & =\frac{\partial^{2} \widehat{J}^{p}}{\partial \tau^{2}}\left(\tau^{p, *}, \sigma^{p, *}\right) \\
& =\left(\widehat{\psi}^{p}\right)^{\prime \prime}\left(\tau^{p, *}\right)-2 \sum_{i=1}^{s} \sum_{j=1}^{s} \frac{\partial \widehat{J}^{p}}{\partial \sigma_{i}^{p}}\left(\tau^{p, *}, \sigma^{p, *}\right) \xi^{\prime}\left(\tau^{p, *}\right)_{i} c_{i, j}\left(\tau^{p, *}, \sigma^{p, *}\right) \xi^{\prime}\left(\tau^{p, *}\right)_{j} .
\end{aligned}
$$

Now, (3.16) says that $\left(\partial^{2} \widehat{J}^{p} / \partial \sigma_{j}^{p} \partial \tau\right)\left(\tau^{p, *}, \sigma^{p, *}\right)=0, j=1, \ldots, s$; which by (3.17), is equal to $-2 \sum_{j=1}^{s} c_{i, j}\left(\tau^{p, *}, \sigma^{p, *}\right) \xi^{\prime}\left(\tau^{p, *}\right)_{j}, i=1, \ldots, s$. Multiplying by $\xi^{\prime}\left(\tau^{p, *}\right)_{i}$ and summing over $i$ we obtain $\left(\widehat{\psi}^{p}\right)^{\prime \prime}\left(\tau^{p, *}\right)=0$.

\section{The solution procedure}

For a fixed positive integer $p$ we here present an algorithm for locating the first zero, $\tau^{p, *}$ of the function $\psi^{p}$ defined by (3.6). It is based on an iteration which is a simple variation of the secant method for locating a zero of a nonlinear function.

The results of Theorem 3.1 imply that $\widehat{\psi}^{p}$ has a triple zero at $\tau=\tau^{p, *}$. Given two starting values $\tau_{1}$ and $\tau_{2}$, the secant method adapted to the case of multiple roots (see [12], page 153) results in the recursive formula

$$
\tau_{k+1}=\tau^{k}+\frac{\tau_{k}-\tau_{k-1}}{\mu_{k}-1}, \quad k=2,3, \ldots,
$$

where

$$
\mu_{k}=\sqrt[3]{\frac{\hat{\psi}^{p}\left(\tau_{k-1}\right)}{\hat{\psi}^{p}\left(\tau_{k}\right)}} .
$$

Of course, in practice we use these formulae with values of the function $\psi^{p}$ as defined by (3.6) rather than $\widehat{\psi}^{p}$. 


\section{Algorithm}

1. Choose a positive integer $p$ (the number of partitions of $[0, T]$ for the control parametrisation). Choose a value for the relative accuracy $\varepsilon$.

2. $\tau_{2}=T$

repeat $\tau_{2}=0.5 * \tau_{2}$; evaluate $\psi^{p}\left(\tau_{2}\right)$ until $\psi^{p}\left(\tau_{2}\right)>0$; $\tau_{1}=0.5 * \tau_{2}$.

3. Evaluate $\psi^{p}\left(\tau_{1}\right)$ and compute $\tau_{3}$ from (4.1) and (4.2); $k=2$.

4. While $\left|\tau_{k+1}-\tau_{k}\right|<\varepsilon T$ do

evaluate $\psi^{p}\left(\tau_{k+1}\right)$

(a) if $\psi^{p}\left(\tau_{k+1}\right)=0$ then repeat

$$
\begin{aligned}
& \tau_{k+1}=\tau_{k+1}-\min \left\{2 \varepsilon, 0.5 *\left(\tau_{k+1}-\tau_{k}\right)\right\} \\
& \text { recompute } \psi^{p}\left(\tau_{k+1}\right)
\end{aligned}
$$

until $\psi^{p}\left(\tau_{k+1}\right)>0$

(b) $k=k+1$; compute $\tau_{k+1}$ from (4.1) and (4.2).

\section{Notes}

1. Step 2 generates two points $\tau_{1}$ and $\tau_{2}$ to the left of $\tau^{p, *}$.

2. The iterative procedure in the algorithm is a combination of the secant method and bisection-like steps which generate a strictly monotonic increasing sequence $\left\{\tau_{k}\right\}$ bounded above by $\tau^{p, *}$. Convergence to $\tau^{p, *}$ can therefore be established if the algorithm is allowed to proceed to the limit $k \rightarrow \infty$.

3. Step 4(a) is included to cover the possibility that $\tau_{k+1}>\tau^{p, *}$. If this occurs, $\tau_{k+1}$ is decreased by amounts no greater than $2 \varepsilon$ until $\tau_{k+1}<\tau^{p, *}$. If this step is invoked then it has the effect of bracketing $\tau^{p, *}$ in an interval of width no more than $2 \varepsilon$. The midpoint of this interval then satisfies the convergence criterion.

4. The iterative formula (4.1) has the same order of convergence as the secant method although, if step 4(a) is invoked, the method is only first order. Experience shows, however, that convergence to a small neighbourhood of $\tau^{p, *}$ is extremely fast and $\tau_{3}$ usually gives a reasonably good estimate of $\tau^{p, *}$. In the examples we tried, an accuracy of less than $1 \%(\varepsilon=0.01)$ was obtained with at most 4 or 5 function evaluations. 
An important issue is the relationship between $\tau^{p, *}$, the solution to the approximate problem, and $\tau^{*}$, the solution to the original problem $(P)$. Convergence results exist $([5,6,11,13])$ for optimal control problems, including minimum time problems, which show that, as $p \rightarrow \infty$, the solution to the approximate problem converges to the solution to the original problem.

\section{Examples}

Here we apply the algorithm of the previous section to estimate the minimum trapping time for two simple problems. Both problems were solved using the optimal control software MISER ([7]).

EXAMPLE 5.1. From [14], the state equations for a continuous stirred-tank chemical reactor are

$$
\begin{aligned}
& \dot{x}_{1}(t)=-2\left[x_{1}(t)+0.25\right]+\left[x_{2}(t)+0.5\right] \exp \left[\frac{25 x_{1}(t)}{x_{1}(t)+2}\right]-\left[x_{1}(t)+0.25\right] u(t) \\
& \dot{x}_{2}(t)=-0.5-x_{2}(t)-\left[x_{2}(t)+0.5\right] \exp \left[\frac{25 x_{1}(t)}{x_{1}(t)+2}\right] .
\end{aligned}
$$

Here $x_{1}(t)$ is the deviation from the steady state temperature, $x_{2}(t)$ is the deviation from the steady state concentration and $u(t)$ is a normalised control variable which represents the effect of coolant flow on the chemical reaction.

Suppose the aim is to return the temperature and concentration from a given initial state to a small neighbourhood of their steady state values as quickly as possible and maintain them there for a given time period. This is a minimum trapping time problem and for the purposes of this example we choose the time period as $[0, T]$ where $T=1$. The initial conditions are

$$
x_{1}(0)=0.05, \quad x_{2}(0)=0
$$

and the bounds on the control are

$$
-1 \leq u(t) \leq 1 .
$$

The aim is to drive the system to the target given by

$$
\|x(t)\| \leq 0.01 \text {. }
$$

Corresponding to this we define the function $g:[0, T] \times R^{2} \rightarrow R$ as

$$
g(t, x)=100\|x(t)\|-1 \leq 0 .
$$

Table 5.1 summarises the results for the solution of this problem for the cases $p=10,20$ and 40 . In these three cases, quite accurate estimates of $\tau^{p, *}$ are obtained with only three evaluations of $\psi^{p}(\tau)$, that is, by executing 
only steps 1 to 3 of the algorithm. The results show that the case $p=20$ probably gives an accurate enough estimate of $\tau^{*}$ (to within, say $1 \%$ ) for most problems. The optimal control $u(t)$, corresponding to the minimum trapping time $\tau^{20, *}$, is plotted Figure 5.1(a) and the corresponding states $x_{1}(t)$ and $x_{2}(t)$ are shown in Figure 5.1(b).

TABLE 5.1. Results for Example 5.1.

\begin{tabular}{|c|c|c|c|c|c|c|c|}
\hline$p$ & $\tau_{1}$ & $\psi\left(\tau_{1}\right)$ & $\tau_{2}$ & $\psi\left(\tau_{2}\right)$ & $\tau_{3}$ & $\psi\left(\tau_{3}\right)$ & $\tau_{4}$ \\
\hline 10 & 0.25 & 6.149 & 0.5 & 0.5423 & 0.701 & $1.573 \times 10^{-8}$ & 0.701 \\
\hline 20 & 0.25 & 6.130 & 0.5 & 0.4556 & 0.681 & $2.454 \times 10^{-7}$ & 0.683 \\
\hline 40 & 0.25 & 6.122 & 0.5 & 0.4483 & 0.680 & $9.317 \times 10^{-7}$ & 0.682 \\
\hline
\end{tabular}

EXAMPLE 5.2. The second example is a simple linear tracking problem from [15], page 223. The system equations are

$$
\begin{aligned}
& \dot{x}_{1}(t)=x_{2}(t) \\
& \dot{x}_{2}(t)=2 x_{1}(t)-x_{2}(t)+u(t)
\end{aligned}
$$

together with the initial conditions

$$
x_{1}(0)=-4.0, \quad x_{2}(0)=0.0 .
$$

Suppose the aim is to drive the state $x_{1}$ close to the trajectory $z(t)=0.2 t$ in minimum time and maintain it there over a given time period. For this example we choose the time period as $[0,6]$ and the bounds on the control are

$$
-10 \leq u(t) \leq 10
$$

The target set is given by $\left|x_{1}(t)-z(t)\right| \leq 0.01$ and corresponding to this we define

$$
g(t, x)=100\left|x_{1}(t)-z(t)\right|-1 \leq 0 .
$$

Table 5.2 summarises the results for the solution of this problem for the cases $p=10,20$ and 40 . In these three cases, quite accurate estimates of $\tau^{p, *}$ are obtained with four or five evaluations of $\psi(\tau)$. Three evaluations are required for step 2 of the algorithm. In the case $p=40$, step 4(a) was executed with $\varepsilon=0.01$ (see the bottom row of Table 5.2). Again the results show that the case $p=20$ probably gives an accurate enough estimate of $\tau^{*}$. The optimal control $u(t)$, corresponding to the minimum trapping time $\tau^{20, *}$, is plotted in Figure 5.2(a) and the corresponding states $x_{1}(t)$ and $x_{2}(t)$ are shown in Figure 5.2(b). 


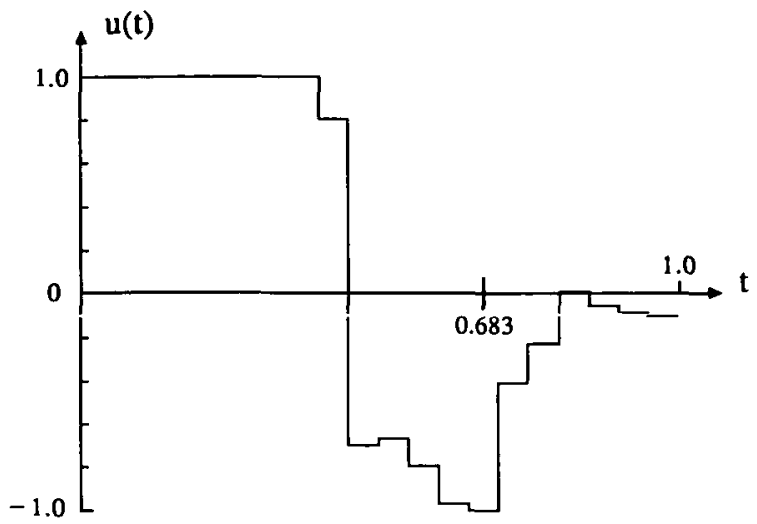

(a)

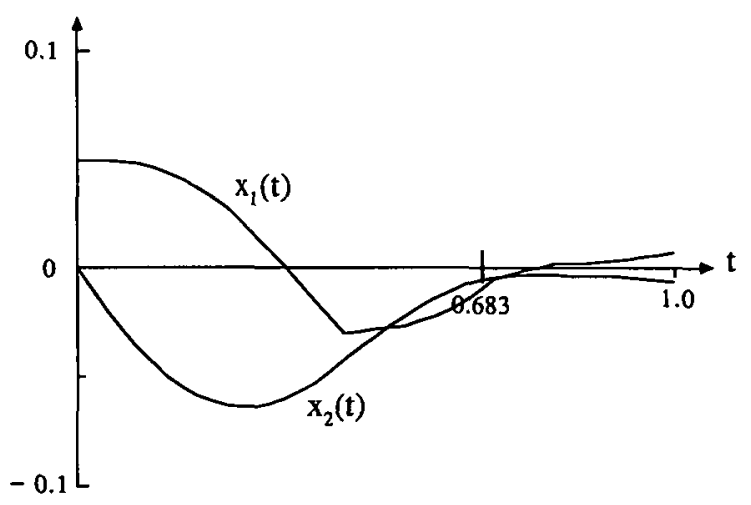

(b)

Figure 5.1. The (a) optimal control and (b) optimal states, for Example 5.1 for the case $p=20$.

TABLE 5.2. Results for Example 5.2.

\begin{tabular}{|c|c|c|c|c|c|c|c|}
\hline$p$ & $\tau_{1}$ & $\psi\left(\tau_{1}\right)$ & $\tau_{2}$ & $\psi\left(\tau_{2}\right)$ & $\tau_{3}$ & $\psi\left(\tau_{3}\right)$ & $\tau_{4}$ \\
\hline 10 & 0.75 & $8.480 \times 10^{4}$ & 1.5 & $1.274 \times 10^{4}$ & 2.35 & 12.76 & 2.45 \\
\hline 20 & 0.75 & $8.442 \times 10^{4}$ & 1.5 & $1.236 \times 10^{4}$ & 2.33 & $4.812 \times 10^{-7}$ & 2.33 \\
\hline 40 & 0.75 & $8.413 \times 10^{4}$ & 1.5 & $1.207 \times 10^{4}$ & 2.32 & 0 & \\
& & & & & 2.20 & 0.2251 & 2.23 \\
\hline
\end{tabular}




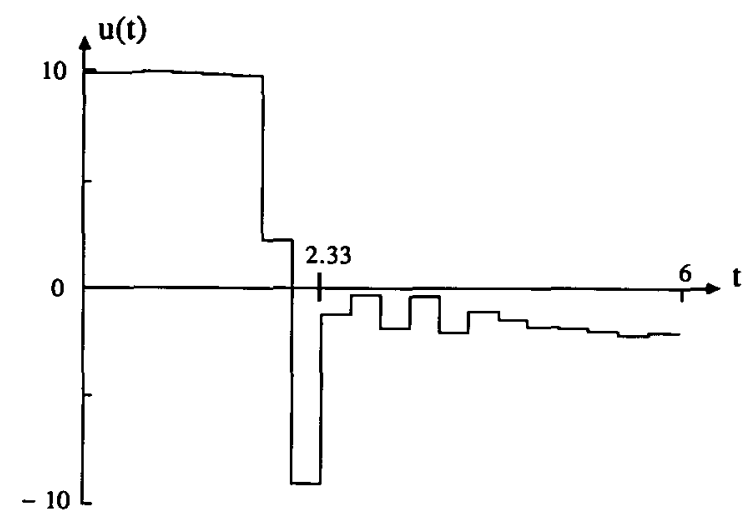

(a)

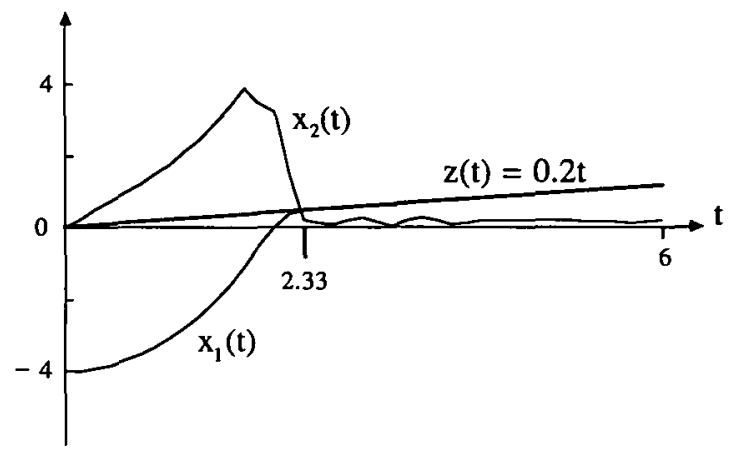

(b)

Figure 5.2. The (a) optimal control and (b) optimal states, for Example 5.2 for the case $p=20$.

\section{Acknowledgement}

This work was partially supported by a research grant provided by the Australian Research Council. 


\section{References}

[1] L. S. Pontyagin, V. G. Boltyanskii, R. V. Gamkrelidze, and E. F. Mishchenko, The Mathematical Theory of Optimal Processes (Wiley 1962).

[2] H. Hermes and J. P. LaSalle, Functional Analysis and Time Optimal Control (Academic Press 1969).

[3] L. Cesari, Optimization Theory and Applications (Springer-Verlag 1983).

[4] N. U. Ahmed, Elements of Finite-Dimensional Systems and Control Theory (Longman Scientific and Technical ! 988).

[5] C. J. Goh and K. L. Teo, "Control parametrization: A unified approach to optimal control problems with general constraints," Automatica 24 (1988) 3-18.

[6] K. L. Teo and L. S. Jennings, "Nonlinear optimal control problems with continuous state inequality constraints," to appear JOTA (1989).

[7] C. J. Goh and K. L. Teo, MISER: An Optimal Control Software (Applied Research Corporation, National University of Singapore, Singapore 1987).

[8] A. Miele, "Gradient algorithms for the optimization of dynamic systems," in C. T. Leondes (ed.), Control and Dynamic Systems: Advances in Theory and Applications (Academic Press 1980) 1-52.

[9] A. Miele and T. Wang, "Primal-dual properties of sequential gradient restoration algorithms for optimal control problems, part 1, basic problems," in F. R. Payne et al. (ed.), Integral Methods in Science and Engineering (Hemisphere Publ. Corp. 1986) 577-607.

[10] A. Miele and T. Wang, "Primal-dual properties of sequential gradient restoration algorithms for optimal control problems, part 2, general problem," J. Math. Analysis and Applic. 119 (1986) 21-54.

[11] K. H. Wong, D. J. Clements, and K. L. Teo, "Optimal control computation for nonlinear time-lag systems," JOTA 47 (1985) 91-107.

[12] J. F. Traub, Iterative Methods for the Solution of Equations (Prentice-Hall 1964).

[13] K. L. Teo and C. C. Lim, "Time optimal control computation with application to ship steering," JOTA 56 (1988) 145-156.

[14] L. Lapidus and R. Luus, "The control of nonlinear systems; part II: Convergence by combined first and second variations," A. I. Ch. E. J. 13 (1967) 108-113.

[15] D. E. Kirk, Optimal Control Theory: An Introduction (Prentice-Hall 1970). 